lexical algorithm based on Mesh keywords associated with each gene. We tested the software's potential by analysing the transcriptional changes induced by the infection of $\mathrm{CD} 4+\mathrm{T}$ cells with the human immunodeficiency virus type 1 (HIV1). Aliquots of either infected or uninfected control cells were obtained at $30 \mathrm{~min}-$ utes, 2, 4, 8, 16, 24, 48 and 72 hours after infection or mock-infection (control) and analysed with the Hu6800

GeneChip. HIV infection of a CD4+ T cell modulated a large number of genes at the transcriptional level. Genes were repressed, induced or modulated significantly very early after inoculation of the virus. Some expected outcomes such as the activation of NFkB, p68 kinase and Rnase L were confirmed by the GeneChip analysis and further corroborated by using real-time quantitative PCR. The modulation of genes and gene families not previously considered associated with HIV infection was also uncovered. Functional genomic approaches represent powerful tools to dissect the relationship between infectious agents and their host cells.

Cossman, Jeffrey

\section{Genome-wide gene expression of the rare, malignant Reed-Stemberg cell of Hodgkin lymphoma}

\author{
Jeffrey Cossman ${ }^{1}$, Joseph Vockley ${ }^{2}$, Kenneth Carter ${ }^{3,4}$, Steven \\ Ruben $^{3}$, Louis Staudt 5 , Steven Barash ${ }^{3}$, Charles Birse ${ }^{3}$, Craig \\ Rosen ${ }^{3}$, Doug Dolginow ${ }^{2} \&$ Greg Lennon ${ }^{2}$
}

\author{
${ }^{1}$ Georgetown University, Washington, DC, USA \\ ${ }^{2}$ GeneLogic, Inc. \\ ${ }^{3}$ Human Genome Sciences, Inc. \\ ${ }^{4}$ International Genetics Associates, Inc \\ ${ }^{5}$ National Cancer Institute, National Institutes of Health, \\ Bethesda, M aryland, USA
}

We have determined the genome-wide gene expression profile of Hodgkin disease, the most common cancer of young adult Americans. Sequences were obtained from approximately 40,000 ESTs (20,000,000 bases) from Hodgkin disease sources. These included cell lines, primary tissue and living, single ReedSternberg cells - the exceedingly rare but biologically active malignant cell of Hodgkin lymphoma. Results of the high throughput sequencing increased the number of named, expressed genes in Hodgkin disease from 100 to 2666. We devised an algorithm to globally compare the gene expression profile of Hodgkin's disease to 100,000 ESTs from different hematopoetic cell types. The analysis supports a B cell lineage of the elusive Reed-Sternberg cell and provides a public database of the gene expression profile of Hodgkin lymphoma ${ }^{1}$ (http://www.hodgkins.georgrtown.edu).

To compare the results of high throughput EST sequencing with microarrays, we are applying Hodgkin expressed genes to Affymetrix arrays containing 42,000 features and representing approximately 30,000 distinct genes. Together, these analyses provide a substantial expressed gene database and a resource for the diagnosis and treatment of Hodgkin disease, other lymphomas and immune system disorders. The comparison addresses the relative informative value of high throughput EST sequencing and expression microarrays in the setting of human cancer.
Dangond, Fernando

\section{Differential gene expression in human spinal cord tissues from ALS patients}

\author{
Femando Dangond ${ }^{1}$, Robert Brown ${ }^{2} \&$ Steven R. Gullans ${ }^{3}$ \\ 'Department of Neurology, Brigham and Women's Hospital, \\ Boston, M assachusetts, USA \\ ${ }^{2}$ Department of Neurology, M assachusetts General Hospital, \\ Boston, M assachusetts, USA \\ ${ }^{3}$ Department of M edicine, Brigham and Women's Hospital, \\ Boston, M assachusetts, USA
}

Amyotrophic Lateral Sclerosis (ALS) is a human neurologic disease characterised by the progressive deterioration of motor function, leading to death within a few years. In some of the familial forms of ALS, a mutation of the superoxide dismutase (SOD) gene has been demonstrated. Recent studies suggest that excitotoxic injury might be responsible for the progressive neuronal degeneration found in ALS spinal cords. In order to investigate differential expression of genes that may be of relevance in ALS pathogenesis, we used the Affymetrix 6800 human DNA array and compared RNA isolated from grey matter of cadaveric tissues, obtained from ALS and non-ALS spinal cords. We discuss our findings and suggest the relative importance of several genes.

de Saizieu, Antoine

\section{Genome-wide transcriptional analysis of bacterial genomes: applications in antibacterial drug discovery}

\author{
Antoine de Saizieu, Hans Gmuender, Christopher Gray \\ $\&$ Wolfgang Keck
}

Hoffmann-La Roche, Infectious Diseases, Bldg 70/139 Grenzacherstrasse124, CH-4070 Basel, Switzerland

An oligonucleotide array of more than 250,000 specific probes representing two complete bacterial genomes including the 1,743 open reading frames of Haemophilus influenzae, and the 1,969 open reading frames of Streptococcus pneumoniae has been designed. When hybridised against labelled genomic DNA, all genes were detected and more than $96 \%$ of the signal intensity values were within a factor of three of the mean. For transcript imaging, microarrays were hybridised against total RNA populations quantitatively represented by labelled cDNAs. The measurements of transcript abundance for all genes were shown to be sensitive, specific, quantitative and reproducible. Nearly $85 \%$ of all S. pneumoniae mRNAs were found expressed during in vitro exponential growth. Identification of regulons important for S. pneumoniae entry into the stationary phase has been used to validate the approach. We will show, for the first time, how a genome-wide transcriptional analysis is applied to bacterial genomes and is being used to identify signal transduction regulatory networks, and to profile antibiotic compounds. 